INFLAMMATORY BOWEL DISEASE

\title{
Dynamics of mucosal permeability and inflammation in collagenous colitis before, during, and after loop ileostomy
} A Münch, J D Söderholm, C Wallon, Å Öst, G Olaison, M Ström

Collagenous colitis has become a more frequent diagnosis but the aetiology of this disease is still unknown. We describe a female patient with intractable collagenous colitis who was treated with a temporary loop ileostomy. She was followed clinically, histopathologically, and functionally by measuring mucosal permeability before surgery, after ileostomy, and after bowel reconstruction. In our case report, active collagenous colitis was combined with increased transcellular and paracellular mucosal permeability. Diversion of the faecal stream decreased inflammation of the mucosa and normalised epithelial degeneration and mucosal permeability. After restoration of bowel continuity, mucosal permeability was altered prior to the appearance of a collagenous layer.

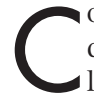
agenous colitis (CC) presents clinically with watery diarrhoea and the diagnosis is confirmed histopathologically by showing a thick subepithelial collagen band and lymphocyte infiltration of the lamina propria and epithelium. The aetiology of this disease is unknown but one hypothesis is that a luminal toxic agent may trigger the onset of CC. ${ }^{1}$ Järnerot et al showed that faecal stream diversion leads to clinical and histopathological remission, although relapse occurred shortly after bowel reconstruction. ${ }^{2}$ Currently there are several therapy options available but only budesonide has been shown to be effective in randomised trials. ${ }^{3}$ The disease can however be resistant to medication and in some patients surgery is the only alternative. We describe a female patient with longstanding socially disabling CC who was followed clinically, histopathologically, and functionally. Biopsies of the sigmoid colon were examined in Ussing chambers to investigate epithelial barrier function. These studies were performed before surgery, after ileostomy for faecal stream diversion, and after bowel reconstruction.

\section{CASE REPORT}

The patient was a 59 year old female nurse with a medical history of coronary heart disease, hypertension, and a pacemaker because of tachy-bradyarrhythmia. She had been unable to work for the past five years because of chronic diarrhoea with watery bowel movements up to 20 times a day. In addition to her regular medication (enalapril, acetylsalicylic acid, furosemide, isosorbide mononitrite, sotalolol, isradipine, estradiol) she had tried nearly all available treatment options for CC (high doses of loperamide, prednisolone, budesonide, 5-aminosalicylic acid, cholestyramine, and norfloxacin) without any major clinical benefit. Because of her desperate situation she was willing to undergo surgery (loop ileostomy) in December 2001. The patient underwent colonoscopy before surgery and microscopic changes typical of CC were present throughout the whole colon, in spite of ongoing budesonide treatment (ceased before operation). Two and four months after loop ileostomy and seven months after bowel reconstruction, multiple biopsies were taken from the same area of the sigmoid colon for special histopathological examination and Ussing chamber studies. Postoperatively, the patient had a wound infection and developed Clostridium difficile colitis following antibiotic treatment. She received metronidazole and improved rapidly. Two separate stool analyses confirmed that she was free of Clostridium difficile infection one month after surgery. The patient improved clinically but developed problems controlling the stoma. In June 2002 the loop ileostomy was closed and bowel continuity restored. The patient started oral medication with budesonide $6 \mathrm{mg}$ daily. At the follow up colonoscopy seven months later the same symptoms as prior to operation had reappeared in spite of budesonide treatment.

\section{METHODS}

\section{Histology}

Biopsies were obtained endoscopically from the colon and stained with haematoxylin-eosin and van Gieson. Specimens from the sigmoid colon were more closely investigated. Two biopsies from the sigmoid colon were taken at each investigation. The degree of surface epithelial cell degeneration was assessed in three arbitrary units. The thickness of the collagenous band was measured in five different areas and the mean value was assessed. ${ }^{4}$ Immunohistochemical staining for CD3 was also performed according to routine procedures. The number of intraepithelial lymphocytes (IEL)/ 100 enterocytes (mean value of three countings) was assessed. Infiltration of mononuclear cells (lymphocytes and plasma cells) in the lamina propria was defined in three arbitrary units. ${ }^{5}$

\section{Ussing chamber}

Biopsies were mounted in modified $1.5 \mathrm{ml}$ Ussing chambers (Grass\&Sweetana, Harvard apparatus Inc., Holliston, Massachusetts, USA) with an exposed tissue area of $1.76 \mathrm{~mm}^{2}$. Continuous oxygenation was given; $\mathrm{pH}$ was kept to 7.4 and temperature to $37^{\circ} \mathrm{C}$. After a 40 minute equilibration period, the marker solution containing chromium-51 ethylenediaminetetraacetate $\left({ }^{51} \mathrm{Cr}\right.$-EDTA) and horseradish peroxidase type VI (HRP) $10 \mu \mathrm{M}$ were added to the mucosal compartment. Every 30 minutes $0.3 \mathrm{ml}$ samples were taken from the serosal side for analysis. ${ }^{51} \mathrm{Cr}$-EDTA uses the paracellular pathway ${ }^{67}$ and HRP permeates mainly transcellularly. ${ }^{8}$ Transepithelial potential difference (Pd), short circuit current (Isc), and transepithelial resistance (TER) were studied for 120 minutes in all specimens. Results

Abbreviations: CC, collagenous colitis; Cr-EDTA, chromiumethylenediaminetetraacetate; $\mathrm{HRP}$, horseradish peroxidase; IBD, inflammatory bowel disease; Isc, short circuit current; Pd, transepithelial potential difference; TER, transepithelial resistance; IEL, intraepithelial ymphocytes 

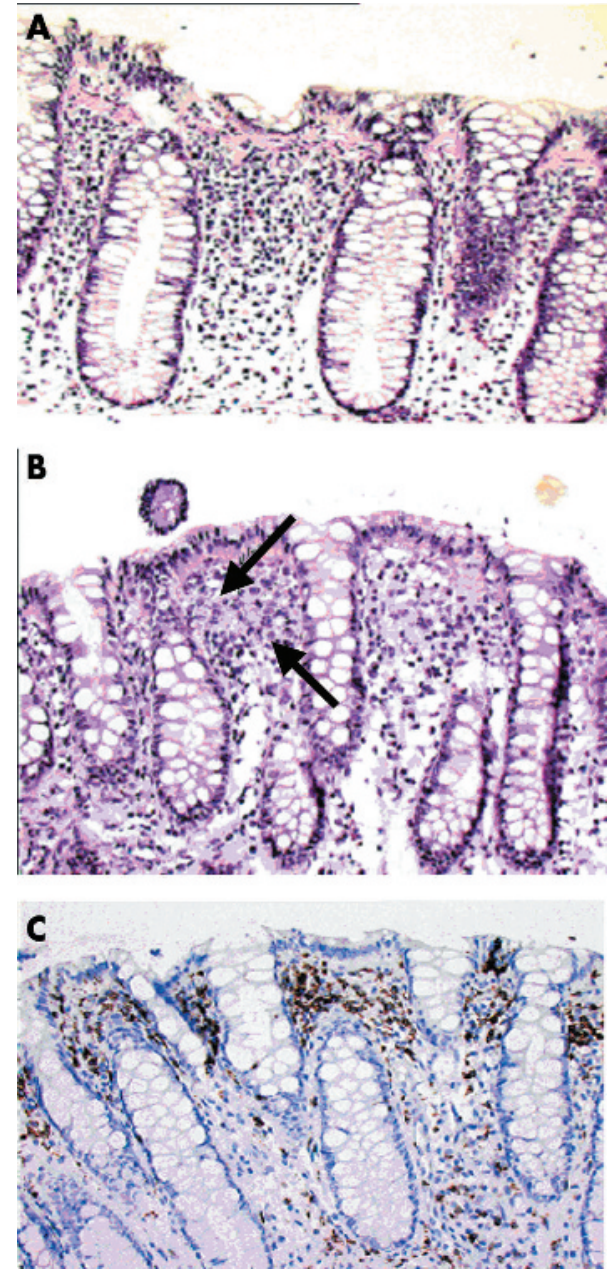

Figure 1 (A) Biopsy from the sigmoid colon prior to operation. Collagen staining (van Gieson). (B) Biopsy from the sigmoid colon two months after loop ileostomy. Collagen staining (van Gieson). Arrows indicate upper and deeper border of the collagen containing layer. The deeper border is diffuse. (C) Biopsy from the sigmoid colon two months after loop ileostomy. Staining for CD3. There were numerous CD3 positive cells in the collagen containing layer.

from a control group of healthy volunteers were used for comparison.

\section{RESULTS}

\section{Histology}

Biopsies taken prior to surgery showed a classical picture of CC. Two months after loop ileostomy the submucosal collagenous layer was reduced in thickness but there was also a remarkable additional infiltration of $\mathrm{CD} 3$ lymphocytes in the collagenous layer. The layer was also indistinct at the deeper border (fig 1). The number of IEL, epithelial degeneration, and the density of the mononuclear cells in the lamina propria decreased (table 1). A further decrease in the collagenous band could be seen after four months and the border became more distinct, but there was still the same grade of density of mononuclear cells in the lamina propria. Upper gastrointestinal endoscopy at that time revealed a normal gastric and duodenal mucosa. Seven months after bowel reconstruction the sigmoid colon presented only with an increase in lymphocytes and plasma cells in the lamina propria whereas the collagenous band was still less than $10 \mu \mathrm{m}$ in the sigmoid colon but increased in thickness in the rest of the colon (data not shown).

\section{Ussing chamber}

Cr-EDTA and HRP sigmoid mucosal permeability were substantially increased before surgery, when the patient had active colitis. Permeability decreased at two months and normalised after four months compared with the control group. Seven months after bowel reconstruction colonic mucosa permeability increased again to a level above the 95th percentile for controls (fig 2). Electrophysiological measurements (Pd, Isc, TER) were stable, indicating viability of all specimens.

\section{DISCUSSION}

Our case report corroborates the findings of Järnerot and colleagues $^{2}$ that faecal stream diversion with a temporary loop ileostomy leads to clinical and histopathological remission in CC. Furthermore, we showed that mucosal barrier dysfunction was present during active CC by measuring permeability in endoscopic biopsies using Ussing chamber technology. During the period with a diverting stoma, repeated biopsies of the sigmoid colon showed that transcellular and paracellular permeability decreased at the same time as inflammation in the lamina propria diminished, and the subepithelial collagenous layer and epithelial degeneration disappeared. Histology after two months showed that the collagenous band was more diffuse and infiltrated by lymphocytes, which could be a result of resolution. We do not believe that this is associated with diversion colitis or an effect of the postoperative Clostridium difficile infection because typical signs of these conditions were not seen histologically.

The present case suggests that active CC may be described as a disease with increased transcellular permeability in addition to increased permeation via tight junctions. The increased permeability seemed however to parallel the degree of epithelial damage. Hypothetically, epithelial cell degeneration could possibly be associated with mucosal barrier dysfunction.. Burgel et al showed that expression of tight junction proteins is diminished in CC but the mucosal architecture in their patients was not much altered compared with normal histology. ${ }^{9}$

Many gastrointestinal diseases have gut barrier dysfunction although it is uncertain if disrupted gut barrier is a primary pathomechanism or secondary to inflammation. ${ }^{10}$ It

Table 1 Time schedule of sigmoid histology

\begin{tabular}{|c|c|c|c|c|}
\hline & $\begin{array}{l}\text { Before } \\
\text { surgery }\end{array}$ & $\begin{array}{l}2 \text { months with } \\
\text { diverting stoma }\end{array}$ & $\begin{array}{l}4 \text { months with } \\
\text { diverting stoma }\end{array}$ & $\begin{array}{l}7 \text { months after } \\
\text { bowel reconstruction }\end{array}$ \\
\hline Epithelial cell degeneration (au 0-3) & 2 & 1 & 0 & 1 \\
\hline Collagenous band thickness $(\mu \mathrm{m})$ & $30(22-38)$ & $25(2-50)$ & $<10$ & $<10$ \\
\hline Intraepithelial lymphocytes (No/100 enterocytes) & $17(13-23)$ & $8(5-11)$ & $14(11-17)$ & $12(9-16)$ \\
\hline $\begin{array}{l}\text { Density of inflammatory (mononuclear cells) in lamina } \\
\text { propria (au 0-3) }\end{array}$ & 2 & 1 & 1 & 2 \\
\hline
\end{tabular}

Values are mean (range) of 3-5 countings.

au, arbitrary units: no $=0$, slight $=1$, moderate $=2$, and heavy $=3$. 

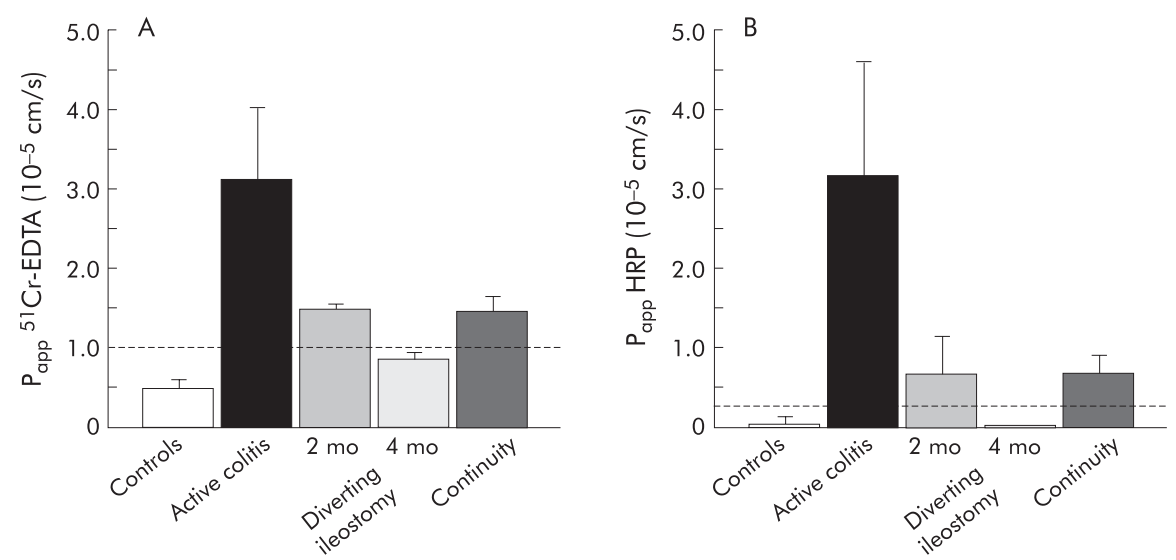

Figure 2 Permeability of chromium-51 ethylenediaminetetraacetate $\left({ }^{51} \mathrm{Cr}\right.$-EDTA) (A) and horseradish peroxidase (HRP) (B) in the sigmoid colon of 19 healthy controls and in the patient with collagenous colitis at different stages of disease (3-5 biopsies of the sigmoid colon were studied at each time point). Permeability is given as the apparent permeability coefficient $\left(P_{\text {app }}\right)$. Values are mean (SEM). Broken line indicates the 95th percentile of controls.

has been suggested that increased mucosal permeability to macromolecules and antigens is a possible initiating event for developing intestinal inflammation in Crohn's disease and ulcerative colitis. ${ }^{67}$ In both diseases however enhanced intestinal permeability is associated with clinical activity and intestinal inflammation, ${ }^{11}$ with tumour necrosis factor $\alpha$ as a possible mediator. ${ }^{12}$ In our case, mucosal permeability was normal four months after faecal stream diversion when histology showed only slight inflammation in the lamina propria, suggesting that the mucosal barrier defect is a consequence of an underlying inflammatory process.

After restoring bowel continuity the patient had a relapse in symptoms, despite continuous budesonide treatment. In parallel, histological findings of CC recurred. In the sigmoid colon mucosal permeability was altered prior to formation of the collagenous band which indicates that the collagenous band is not a primary factor for mucosal barrier defect.

The cause of CC is not known. As faecal stream diversion leads to remission it seems most likely that epithelial damage is caused by a hitherto unknown luminal agent(s) as a first event leading to mucosal inflammation and abnormal permeability. Experimental studies of rabbit and rat colon mucosa have shown that bile salts can alter the integrity of tight junctions. ${ }^{13}$ As bile acid malabsorption is common in $\mathrm{CC},{ }^{14}$ hypothetically bile acids could contribute to disturbed mucosal permeability. Bile acid binders often lead to clinical improvement but do not necessarily correlate with histological remission..$^{15}$ Unfortunately, our patient did not improve when treated with cholestyramine.

In summary, our case of active CC was associated with increased transcellular and paracellular mucosal permeability. Diversion of the faecal stream decreased inflammation of the mucosa and normalised permeability. After restoration of bowel continuity permeability was altered prior to the appearance of a collagenous layer. Further studies are necessary to elucidate the role of increased macromolecular permeability in the pathogenesis of collagenous colitis.

\section{Authors' affiliations}

A Münch, M Ström, Department of Gastroenterology, UHL, Linköping, Sweden
J D Söderholm, C Wallon, G Olaison, Department of Surgery, UHL, Linköping, Sweden

Å Öst, Medilab, Täby, Sweden

Conflict of interest: None declared.

Correspondence to: $\operatorname{Dr} M$ Ström, Department of Gastroenterology, Linköping University Hospital, SE-58185 Linköping, Sweden;

Magnus.Strom@lio.se

Revised version received 18 March 2005

Accepted for publication 23 March 2005

\section{REFERENCES}

1 Bohr J. A review of collagenous colitis. Scand J Gastroenterol 1998;33:2-9.

2 Järnerot G, Tysk C, Bohr J, et al. Collagenous colitis and fecal stream diversion. Gastroenterology 1995;109:449-55.

3 Chande N, Mc Donald JW, Mac Donald JK. Cochrane Database Syst Rev 2003;(1):CD 003575

4 Bogomoletz WV. Collagenous, microscopic and lymphocytic colitis. An elvolving concept. Virchows Archiv 1994;424:573-9.

5 Geboes K, Ridell R, Öst $\AA$, et al. A reproducible grading scale for histological assessment of inflammation in ulcerative colitis. Gut 2000;47:404-9.

6 Söderholm JD, Olaison G, Peterson KH, et al. Augmented increase in tight junction permeability by luminal stimuli in the non-inflamed ileum of Crohn's disease. Gut 2002;50:307-13.

7 Schmitz H, Barmeyer C, Fromm M, et al. Altered tight junction structure contributes to the impaired epithelial barrier function in ulcerative colitis. Gastroenterology 1999;1 16:301-9.

8 Schurmann G, Bruwer M, Klotz A, et al. Transepithelial transport processes at the intestinal mucosa in inflammatory bowel disease. Int $J$ Colorectal Dis 1999; 14:41-6.

9 Burgel N, Bojarski C, Mankertz J, et al. Mechanisms of diarrhea in collagenous colitis. Gastroenterology 2002;123:433-43.

10 DeMeo MT, Mutlu EA, Keshavarzian A, et al. Intestinal permeation and gastrointestinal disease. J Clin Gastroenterol 2002;34:385-96.

11 Jenkins RT, Jones DB, Goodacre C, et al. Reversbility of increased intestinal permeability to $51 \mathrm{Cr}$-EDTA in patients with gastrointestinal inflammatory disease. Am J Gastroenterol 1987;82:1159-64.

12 Söderholm JD, Streutker C, Yang PC, et al. Increased epithelial uptake of protein antigens in the ileum of Crohn's disease mediated by tumour necrosis factor alpha. Gut 2004;53:1817-24.

13 Freel RW, Hatch M, Earnest DL, et al. Role of tight-junctional pathway in bile salt-induced increase colonic permeability. Am J Physiol 1983;245:816-23.

14 Ung KA, Gillberg R, Kilander A, et al. Role of bile acids and bile binding agents in patients with collagenous colitis. Gut 2000;46:170-5

15 Ung KA, Kilander A, Nilsson O, et al. Long-term course in collagenous colitis and the impact of bile acid malabsorption and bile acid sequestrants on histopathology an clinical features. Scand J Gastroenterol 2001;36:601-9. 